\title{
Konfliktregulierung im Umfeld frühneuzeitlicher Strafgerichte: Das Konzept der Infrajustiz in der historischen Kriminalitätsforschung
}

\begin{abstract}
Summary
The article resumes the concept of infrajustice, in particular developed by French historians and adopted to some extent by the Early Modern History of Crime and Criminal Justice. Infrajustice describes alternatives to public criminal justice and extrajudicial practices of mediation, arbitration and alternative dispute resolution, which, nevertheless, were related to some extent to the sphere of public criminal law and justice. Based on a systematic analyses of types of conflicts and crimes, actors and institutions, modes and procedures, and the norms and functions of infrajustice the article observes scope, advantages and deficiencies of the concept. Though infrajustice needs further theoretical development it, nevertheless, provides valuable approaches to "Extrajudicial and Judicial Conflict Resolution" by emphasising the complex relations between the judicial and extrajudicial spheres and the various modes of conflict regulation between social agreement, mediation, arbitration, and punishment.
\end{abstract}

\section{Résumé}

Le présent article propose de revenir sur le concept de l'infrajustice, développé en particulier par les historiens français et adopté, dans une certaine mesure, par l'Histoire du crime et de la justice pénale du début de l'époque moderne. L'infrajustice se rapporte aux solutions autres que la justice pénale publique et pratiques extrajudiciaires telles que la médiation, l'arbitrage et les autres modes de résolution des conflits, qui, toutefois, relèvent partiellement de la sphère de la justice et du droit pénaux publics. En se fondant sur des analyses systématiques des types de conflits et de crimes, des acteurs et des institutions, des modalités et des procédures, ainsi que des normes et des fonctions de l'infrajustice, l'article observe la portée, les avantages et les carences de ce concept. Bien que l'infrajustice doive encore faire l'objet de développements théoriques supplémentaires, elle offre néanmoins des pistes intéressantes en regard de la "résolution extrajudiciaire et judiciaire de conflits », en insistant sur les relations complexes entre les sphères judiciaire et extrajudiciaire, de même que sur les différentes procédures de règlement des conflits telles que l'accord social, la médiation, l'arbitrage et la sanction.

\section{Zum Konzept der Infrajustiz}

Stärker als die Strafrechtsgeschichte hat die historische Kriminalitätsforschung den Blick auf Konfliktregulierung zwischen „Privaten“ im Umfeld der Strafjustiz gerichtet 
und beobachtet, dass Verbrechen und die diesen zugrunde liegenden oder damit einhergehenden Konflikte ,außergerichtlich“ bzw. außerhalb der Justizinstitutionen oder im Zusammenspiel zwischen gerichtlichen und außergerichtlichen Akteuren reguliert wurden. ${ }^{1}$ Solche Phänomene thematisieren zahlreiche Fallstudien zu Kriminalität, Strafjustiz und Konflikt, die räumlich sowohl die größeren Städte als auch den ländlichen Raum behandeln und zeitlich auf das späte Mittelalter und die Frühe Neuzeit fokussieren. ${ }^{2}$ Auch die Rechtsgeschichte hat Phänomene außergerichtlicher ,infrajustizieller“ Konfliktregulierung behandelt, sich allerdings überwiegend auf die neuere Geschichte, einzelne Rechtsgebiete wie vor allem das Arbeits- und Verwaltungsrecht oder spezifische Institutionen wie z.B. den außergerichtlichen Vergleich oder den „Täter-OpferAusgleich“ konzentriert. ${ }^{3}$ Um ,Alternativen zur obrigkeitlichen Rechtsprechung“, ,gesellschaftliche Praxis der Konfliktregulierung “ und ,außergerichtliche Spielräume“ der „Nichtjustiz“ theoretisch zu beschreiben, ${ }^{4}$ hat die französische Forschung seit den 1980er Jahren den Begriff L'infrajudiciare bzw. Infrajustice verwendet, und Benoit Garnot widmete ihr 1995 ein Kolloquium mit zahlreichen Beiträgen zu anderen euro-

1 Zum Forschungsstand: Gerd Schwerhoff, Historische Kriminalitätsforschung, Frankfurt a.M./ New York 2011, hier besonders S. 81-83; Benoît Garnot, Crime et justice aux XVIIe et XVIIIe siècles, Paris 2000; Benoît Garnot, Justice et société en France au XVIe, XVIIe et XVIIIe siècles, Paris 2000; Leonida Tedoldi, La spada e la bilancia. La giustizia penale nell'Europa moderna (secc. XVI - XVIII), Rom 2008; Xavier Rousseaux/René Lévy (Hrsg.), Le penal dans tous ses Etats. Justice, Etats et sociétés en Europe (XII ${ }^{\mathrm{e}}-\mathrm{XX}{ }^{\mathrm{e}}$ siècles), Brüssel 1997; Andreas Blauert/Gerd Schwerhoff(Hrsg.), Kriminalitätsgeschichte. Beiträge zur Sozial- und Kulturgeschichte der Vormoderne, Konstanz 2000; Marco Bellabarba/Gerd Schwerhoff/Andrea Zorzi (Hrsg.), Criminalità e giustizia in Germania e in Italia. Pratiche giudiziarie e linguaggi giuridici tra tardo Medioevo ed età moderna/Kriminalität und Justiz in Deutschland und Italien. Rechtspraktiken und gerichtliche Diskurse in Spätmittelalter und Früher Neuzeit, Bologna/Berlin 2001; Rebekka Habermas/Gerd Schwerhoff (Hrsg.), Verbrechen im Blick. Perspektiven der neuzeitlichen Kriminalitätsgeschichte, Frankfurt a.M./New York 2009.

2 Vgl. exemplarisch: Peter Schuster, Eine Stadt vor Gericht. Recht und Alltag im spätmittelalterlichen Konstanz, Paderborn 1999; Harriet Rudolph, „Eine gelinde Regierungsart“. Peinliche Strafjustiz im geistlichen Territorium. Das Hochstift Osnabrück (1716-1803), Konstanz 2001; Joachim Eibach, Frankfurter Verhöre. Städtische Lebenswelten und Kriminalität im 18. Jahrhundert, Paderborn u.a. 2003; Karl Härter, Policey und Strafjustiz in Kurmainz. Gesetzgebung, Normdurchsetzung und Sozialkontrolle im frühneuzeitlichen Territorialstaat, Frankfurt a.M. 2005; Hervé Piant, Une justice ordinaire. Justice civile et criminelle dans la prévôté royale de Vaucouleurs sous l'Ancien Régime, Rennes 2006; Stefan Esders (Hrsg.), Rechtsverständnis und Konfliktbewältigung. Gerichtliche und außergerichtliche Strategien im Mittelalter, Köln u.a. 2007; Benoît Garnot (Hrsg.), Normes juridiques et pratiques judiciaires du Moyen Âge à l'époque contemporaine, Dijon 2007.

3 Rainer Maria Kiesow/Dieter Simon (Hrsg.), Vorzimmer des Rechts, Frankfurt a.M. 2006; Christoph A. Stumpf, Alternative Streitbeilegung im Verwaltungsrecht. Schiedsgerichtsbarkeit - Schiedsgutachten - Mediation - Schlichtung, Tübingen 2006; Britta Schubel, Geschichte und Gegenwart außergerichtlicher Erledigung von Strafsachen durch ehrenamtliche Schiedsinstanzen in den neuen Bundesländern, Berlin 1997.

4 Francisca Loetz, L'infrajudiciaire. Facetten und Bedeutung eines Konzepts, in: Blauert/ Schwerhoff, Kriminalitätsgeschichte, S. 545-562. 
päischen Ländern. ${ }^{5}$ Seitdem hat die historische Kriminalitätsforschung in Frankreich, Deutschland, Italien und Spanien das Konzept rezipiert und in einigen empirischen Fallstudien angewandt sowie versucht, es theoretisch weiterzuentwickeln und zu modifizieren. ${ }^{6}$

Das obrigkeitlich-staatliche Justizsystem - die justice publique - fungiert dabei als Bezugsrahmen, an dessen Rändern oder in dessen Vorhof private außergerichtliche Konfliktregulierung in der Sphäre der Infrajustiz oder justice privée stattfand, die durch harmonie, jugement en équité, médiation, réparation et non la répression gekennzeichnet seien. ${ }^{7}$ Die beiden Sphären sind allerdings kaum scharf zu trennen und verweisen auf den vormodernen Rechtspluralismus, der ein wichtiges Element des Konzepts „Infrajustiz" bildet. Denn die vormodernen Rechtssysteme in Europa verfügten über keine völlig verstaatlichte und professionalisierte Justiz, sondern eine Vielzahl von Institutionen, Organen und Akteuren befasste sich mit kriminellem/deviantem Verhalten und den entsprechenden Konflikten: obrigkeitliche niedere und höhere Gerichte, Frevel- und Rügegerichte, Stadt- und Dorfgerichte, städtische und genossenschaftliche Organe, kirchliche Instanzen, Regierungen, juristische Fakultäten, oder Herrscher bzw. Inhaber von Gerichtsrechten, die mittels Urteilsbestätigung oder Gnadengewährung eingebunden waren. ${ }^{8}$ Besonders im lokalen Bereich lassen sich staatliche kaum von Gerichts-

5 Zuerst: Alfred Soman, L'infra-justice à Paris d'après les archives notariales, in: Histoire, économie et société 3 (1982), S. 369-375; weiterhin grundlegend zum Konzept: Benoît Garnot (Hrsg.), L'infrajudiciaire du Moyen Âge à l'époque contemporaine. Actes du colloque de Dijon, 5 - 6 octobre 1995, Dijon 1996; Jean-François Leclerc, Justice et infra-justice en NouvelleFrance. Les voies de fait à Montréal entre 1700 et 1760, in: Criminologie 18 (1985), S. 25-39; Jean-Claude Farcy, Justice privée et Justice publique. Approches de l'historiographie (France, XVIIIe-XXe siècles), in: La résolution des conflits. Justice privée et Justice publique, une frontière mouvante, Centre d'Histoire Judiciaire UMR 8025 du CNRS, Lille 2, 2011 [online: http://chj-cnrs.univ-lille2.fr/spip.php?article429].

6 Benoît Garnot, Justice, infrajustice, parajustice et extra justice dans la France d'Ancien Régime, in: Crime, Histoire \& Sociétés/Crime, History \& Societies 4 (2000), S. 103-120; Loetz, L'infrajudiciaire; Tomás A. Mantecón Movellán, El peso de la infrajudicialidad en el control del crimen durante la Edad Moderna, in: Estudis 28 (2002), S. 43-75; Andrea Zorzi, Conflits et pratiques infrajudiciaires dans les formations politiques italiennes du XIIIe au XVe siècle, in: Garnot, L'infrajudiciaire, S. 19-36; Marco Bellabarba, La représentation des délits entre droit public et droit privé. L'infrajustice dans les criminalistes italiens de l'époque moderne (XVe XVIIe siècle), in: Garnot, L'infrajudiciaire, S. 55-67; Andrea Zorzi, Negoziazione penale, legittimazione giuridica e poteri urbani nell'Italia comunale, in: Bellabarba/Schwerhoff/Zorzi, Criminalità, S. 13-34; Massimo Della Misericordia, La mediazione giudiziaria dei conflitti sociali alla fine del medioevo. Tribunali ecclesiastici e resistenza comunitaria in Valtellina, in: Bellabarba/Schwerhoff/Zorzi, Criminalità, S. 135-171; Marco Bellabarba, Pace pubblica e pace privata: linguaggi e istituzioni processuali nell'Italia moderna, in: Bellabarba/Schwerhoff/ Zorzi, Criminalità, S. 189-213; Jacques Chiffoleau/Claude Gauvard/Andrea Zorzi (Hrsg.), Pratiques sociales et politiques judiciaires dans les villes de l'Occident à la fin du Moyen Âge, Rom 2007.

7 Farcy, Justice privée et Justice publique, S. 15.

8 Harriet Rudolph/Helga Schnabel-Schüle (Hrsg.), Justiz = Justice = Justicia? Rahmenbedingungen von Strafjustiz im frühneuzeitlichen Europa, Trier 2003; Giovanni Chiodi/Claudio Povolo (Hrsg.), L' amministrazione della giustizia penale nella Repubblica di Venezia, 2 Bde., Sommacampagna (Verona) 2004; Benoît Garnot, Histoire de la justice. France, XVIe - XXIe siècle, Paris 2009. 
barkeiten anderer, intermediärer Gewalten (Kirche, Genossenschaften, Kommunen, Zünfte usw.) trennen, zumal sich Privat-, Ordnungs- und Strafrecht bzw. Konfliktlagen wie „Ehre und Gewalt“, „Frevel/Ordnungsverstöße“ oder „Sexualität/Ehe/Familie“ vielfach überschnitten. An der Konfliktbearbeitung waren obrigkeitlich-staatliche Gerichte, intermediäre Gewalten, lokale Gemeinschaften, Amtspersonen, Nachbarschaften, Familien und sonstige Private beteiligt, die sich in einem weiten infrajustiziellen Raum bewegten. ${ }^{9}$ Eine exakte Unterscheidung von ,außergerichtlich - gerichtlich“ ist folglich für das vormoderne Europa schwierig, und Infrajustiz eignet sich damit auch als kritischer Ausgangspunkt, um das Begriffspaar außergerichtliche und gerichtliche Konfliktregulierung zu präziseren und eine exkludierende Dichotomie eines „EntwederOder" - entweder Gericht oder eine außergerichtliche Form - zu überwinden.

Auch das Konzept der Infrajustiz setzt außergerichtliche Konfliktregulierung in Beziehung zum Rechtssystem, siedelt sie in dessen Umfeld oder an dessen Rand an und beobachtet das Spannungsverhältnis und die Interaktionen zwischen den beiden Sphären. Infrajustiz wird damit als eine Art Übergangszone zwischen staatlicher Justiz und informeller Autoregulation sozialer Konflikte konzeptualisiert. In dieser erfolgt die Konfliktbearbeitung zwischen Privaten mit Hilfe Dritter und eher informeller, vermittelnder Modi, aber auch durch intermediäre Gewalten oder im Zusammenspiel mit obrigkeitlich-staatlichen Gerichten, wobei Akteure in unterschiedlichen Rollen agieren konnten. Infrajustiz behält folglich das Rechtssystem als Bezugspunkt, erweitert jedoch das Feld im Hinblick auf die Konflikte/Verbrechen, die Akteure, die Verfahren, Kommunikationen und Interaktionen sowie die Funktionen und Zwecke, die über Strafe/ Sanktion hinausgehen und Schlichtung, Kompensation, Interessenausgleich oder Aushandlungsprozesse mit einbeziehen. Die Quellenbasis für die Rekonstruktion infrajustizieller Praktiken ist freilich begrenzt, da sich autonome soziale Konfliktregulierung kaum in überlieferten schriftlichen Zeugnissen niedergeschlagen hat und Infrajustiz letztlich aus Quellen rekonstruiert werden muss, die mehr oder weniger der obrigkeitlichen Sphäre zuzurechnen sind (Gerichts- und Verwaltungsakten, Notariatsakten, Eingaben, Gesuche, Bittschriften usw.). Ausgehend von diesen Grundzügen soll im Folgenden das Modell der Infrajustiz - beschränkt auf den Bereich der Strafrechtsgeschichte/historischen Kriminalitätsforschung - systematischer beschrieben werden für:

- die Konfliktbereiche und das damit verbundene deviante/kriminelle Verhalten;

- die Akteure und Institutionen der Infrajustiz;

- die Praktiken, Verfahren oder Modi der infrajustiziellen Konfliktregulierung;

- die Normen und Ordnungsmodelle sowie die Funktionen der infrajustiziellen Konfliktregulierung.

9 Jenny Thauer, Gerichtspraxis in der ländlichen Gesellschaft. Eine mikrohistorische Untersuchung am Beispiel eines altmärkischen Patrimonialgerichts um 1700, Berlin 2001; Magnus Eriksson/Barbara Krug-Richter (Hrsg.), Streitkulturen. Gewalt, Konflikt und Kommunikation in der ländlichen Gesellschaft der frühen Neuzeit, Köln 2003; Barbara Krug-Richter/Ruth-E. Mohrmann (Hrsg.), Praktiken des Konfliktaustrags in der Frühen Neuzeit, Münster 2004; Julia Haack, Der vergällte Alltag. Zur Streitkultur im 18. Jahrhundert, Köln u.a. 2008. 


\section{Konfliktbereiche und Kriminalität}

Im Mittelpunkt der Infrajustiz stehen deviante/kriminelle Handlungen bzw. Konflikte zwischen ,privaten“ Konfliktparteien: die conflits interindividuels. Dabei handelt es sich vorwiegend um Ehrkonflikte, interpersonale Gewalt wie Schlägereien, Körperverletzungen und Totschlag, familiäre und Ehekonflikte, sexuelle Devianz (Unzucht, Ehebruch), Konflikte und Vergehen im ländlichen Bereich sowie ökonomische Konflikte wie vor allem Verschuldung oder Bankrott. ${ }^{10}$ Besondere Bedeutung wird den Gewaltund Ehrkonflikten beigemessen, da sich Ehre und Reputation über infrajustizielle Mechanismen besser bewahren oder Kompensationen aushandeln ließen als über die staatliche Strafjustiz, von der insgesamt eine infamierende Wirkung ausgehen konnte. ${ }^{11}$ Konflikte zwischen Privaten und dem Staat bzw. der Obrigkeit werden dagegen kaum systematisch einbezogen, weil der Staat diese in der Regel als Normverstöße/Verbrechen interpretierte und meist mit einem Strafverfahren bzw. Strafe reagierte. Schwere Verbrechen oder solche, bei denen Obrigkeit und Staat ein besonderes Verfolgungsund Strafinteresse entwickelten wie bei schwerer Eigentumskriminalität, der Verfolgung von Diebes- und Räuberbanden, politischen Verbrechen oder im Bereich der öffentlichen Sicherheit, finden daher kaum Berücksichtigung bzw. diesbezüglich lassen sich keine infrajustiziellen Praktiken nachweisen.

Dagegen lassen sich bei geringeren Vergehen, Policeydelikten, Freveln, Gewalt- und Ehrkonflikten sowie Konflikten und Kriminalität in den Bereichen Familie, Ehe und Sexualität „,infrajustizielle Praktiken“ und außergerichtliche Akteure auch im Kontext der obrigkeitlichen Strafjustiz feststellen. Dabei handelt es sich z.B. um Bitten an die Obrigkeit um Disziplinierung und Bestrafung von Familienangehörigen (anstelle einer formellen Anzeige), das Aushandeln von Strafen und Gnade oder die Mediation von Opferentschädigung mittels der Lettres de cachet, Supplikationen oder Gnadenbitten. ${ }^{12}$

10 Vgl. die zahlreichen Beiträge in Garnot, L'infrajudiciaire sowie Benoît Garnot (Hrsg.), La petite délinquance du Moyen Age à l'époque contemporaine. Actes du colloque de Dijon 9 \& 10 octobre 1997, Dijon 1998; Michael Frank, Dörfliche Gesellschaft und Kriminalität. Das Fallbeispiel Lippe 1650-1800, Paderborn u.a. 1995; Christine Schedensack, Formen der auBergerichtlichen gütlichen Konfliktbeilegung, Vermittlung und Schlichtung am Beispiel nachbarrechtlicher Konflikte in Münster (1600-1650), in: Westfälische Forschungen 47 (1997), S. 643-667; Christine Schedensack, Nachbarn im Konflikt. Zur Entstehung und Beilegung von Rechtsstreitigkeiten um Haus und Hof im frühneuzeitlichen Münster, Münster 2007; Gerhard Sälter, Lokale Ordnung und soziale Kontrolle in der frühen Neuzeit. Zur außergerichtlichen Konfliktregulierung in einem kultur- und sozialhistorischen Kontext, in: Kriminologisches Journal 32 (2000), S. 19-42.

11 Martin Dinges, Négocier son honneur dans le peuple parisien au XVIIIe siècle: la rue, ,l'infrajudiciaire" et la justice, in: Garnot, L'infrajudiciaire, S. 393-404; Martin Dinges, Der Maurermeister und der Finanzrichter. Ehre, Geld und soziale Kontrolle im Paris des 18. Jahrhunderts, Göttingen 1994; Klaus Schreiner/Gerd Schwerhoff (Hrsg.), Verletzte Ehre. Ehrkonflikte in Gesellschaften des Mittelalters und der Frühen Neuzeit, Köln u.a. 1995.

12 Arlette Farge/Michel Foucault, Familiäre Konflikte. Die „Lettres de cachet“. Aus den Archiven der Bastille im 18. Jahrhundert, Frankfurt a.M. 1989; Cecilia Nubola/Andreas Würgler (Hrsg.), Suppliche e „gravamina“. Politica, amministrazione, guistizia in Europa (secoli XVXVIII), Bologna 2002; Karl Härter/Cecilia Nubola (Hrsg.), Grazia e giustizia. Figure della clemenza fra tardo medioevo ed età contemporanea, Bologna 2011; Jean-Luc Chabot/Stéphane Gal/Christophe Tournu (Hrsg.), Figures de la médiation et lien social, Paris u.a. 2006. 
Carl Hoffmann hat z.B. für das Augsburg des 16. Jahrhunderts und die Konfliktfelder Gewalt und Totschlag, Beleidigungen, Verschuldung und Bankrott, Ehestreit, Ungehorsam gegen den Hausvater und „Übelhausen“ gezeigt, dass private Einigungsverfahren Teil des öffentlichen Strafverfahrens waren und Auswirkungen auf die Strafpraxis hatten. ${ }^{13}$ Bei minderschweren Delikten wie Körperverletzungen und Schlägereien waren die infrajustiziellen Spielräume und die Mitwirkung von Dritten besonders ausgeprägt, denn die Obrigkeit maß der außergerichtlichen Konfliktregulierung durch Kompensation und Schlichtung besondere Bedeutung für die Bewahrung des Friedens und der guten Ordnung bei. ${ }^{14}$ Martin Dinges hat solche infrajustiziellen Praktiken auch für Ehrkonflikte im frühneuzeitlichen Paris aufgezeigt, und ich selbst habe Justiznutzung und Konfliktregulierung im Zusammenspiel gerichtlicher und außergerichtlicher Praktiken am Beispiel von Unzuchtsverfahren und Konflikten um den Unterhalt außerehelich geborener Kinder für das frühneuzeitliche Kurmainz dargestellt. ${ }^{15}$

\section{Akteure}

Im Konzept der Infrajustiz kommt neben den Konfliktparteien Dritten eine wesentliche Bedeutung zu, die als Mediatoren, Arbiter, Schlichter oder auch Verbindungsglieder zur Obrigkeit fungierten; sie sind die zentralen Akteure, die Funktionen wie Schlichten, Vermitteln und auch Entscheiden ausübten. Systematisiert man die Forschung, ${ }^{16}$ lassen sich idealtypisch drei Gruppen unterscheiden:

13 Carl A. Hoffmann, Außergerichtliche Einigung bei Straftaten als vertikale und horizontale soziale Kontrolle im 16. Jahrhundert, in: Blauert/Schwerhoff, Kriminalitätsgeschichte, S. 563-579.

14 Martin Scheutz, Zwischen Schlägen und gerichtlichem Ausgleich. Formen der Konfliktaustragung in niederösterreichischen Gerichtsakten des 18. Jahrhunderts, in: Krug-Richter/Mohrmann, Praktiken des Konfliktaustrags, S. 169-186; zusammenfassend hierzu demnächst: Karl Härter, Violent Crimes and Retaliation in the European Criminal Justice System between the Seventeenth and Nineteenth Century, in: Günther Schlee/Bertram Turner (Hrsg.), On Retaliation [im Druck].

15 Dinges, Négocier son honneur; Dinges, Maurermeister; Karl Härter, Erfahrungen in der frühneuzeitlichen Strafjustiz, in: Paul Münch (Hrsg.), „Erfahrung” als Kategorie der Frühneuzeitgeschichte, München 2001, S. 377-388; Karl Härter, Kriminalität und Praxis der Strafjustiz im geistlichen Territorialstaat des Alten Reiches: Sexuelle Delinquenz und Justiznutzung im frühneuzeitlichen Kurmainz, in: Bellabarba/Schwerhoff/Zorzi, Criminalità, S. 101-134.

16 Vgl. insgesamt die zahlreichen Beiträge hierzu in Garnot, L'infrajudiciaire, S. 197-357; sowie Garnot, Justice, infrajustice, parajustice, S. 111 ff.; Benoit Garnot (Hrsg.), Juges, notaires et policiers délinquants XIV. - XX. Siècle, Dijon 1997; Stefan Brakensiek/Heide Wunder (Hrsg.), Ergebene Diener ihrer Herren? Herrschaftsvermittlung im alten Europa, Köln u.a. 2005; Ulinka Rublack, Frühneuzeitliche Staatlichkeit und lokale Herrschaftspraxis in Württemberg, in: Zeitschrift für Historische Forschung 24 (1997) S. 347-376; Stefan Brakensiek, Fürstendiener - Staatsbeamte - Bürger. Amtsführung und Lebenswelt der Ortsbeamten in niederhessischen Kleinstädten (1750-1830), Göttingen 1999; André Holenstein/Frank Konersmann/Josef Pauser/Gerhard Sälter (Hrsg.), Policey in lokalen Räumen. Ordnungskräfte und Sicherheitspersonal in Gemeinden und Territorien vom Spätmittelalter bis zum frühen 19. Jahrhundert, Frankfurt a.M. 2002. 
1. Soziale Gemeinschaften, Nachbarschaften, Familien, Kollegen, Freunde oder Zeugen. Insgesamt gehören diese Akteure freilich weitgehend dem Bereich der autonomen sozialen Konfliktregulierung an, der sich nur schwer in den Quellen fassen lässt.

2. Amtsträger, lokale Amtleute, Vögte, Stadtviertelmeister, Angehörige kommunaler oder ländlicher Polizei-, Sicherheits- und Ordnungskräfte, Schreiber, Notare und Anwälte, Geistliche und Pfarrer sowie Funktions- und Amtsträger von Kommunen, Genossenschaften und Korporationen.

3. Justizpersonal und sonstige Herrschafts-, Amts- und Funktionsträger, die auch in ihren offiziellen Funktionen infrajustizielle Praktiken anwenden konnten: Richter und Schöffen übernahmen Mediatorenfunktionen; Gerichtsschreiber fertigten außergerichtliche Schriftstücke an (z.B. Supplikationen) oder Herrscher gewährten außerhalb des ordentlichen Verfahrens in einem Aushandlungsprozess Strafminderung und Gnade.

Die Forschung hat vor allem die Bedeutung der zweiten Gruppe - der im infrajustiziellen Raum handelnden Amtsträger - betont, denen die folgenden Qualitäten/Merkmale zukommen: allgemeines Ansehen, Sozialprestige bzw. soziales Kapital und persönlicher Einfluss; sehr gute Integration in die jeweiligen sozialen Gemeinschaften bzw. sozialen Milieus, dem Vermittler und Konfliktparteien angehören; ein ausdifferenziertes Beziehungsnetzwerk (réseau de relations); spezifische Qualitäten und rechtliche Kenntnisse als Notare, Schreiber oder Geistliche wie Schreibfähigkeit, Beherrschung der obrigkeitlich-rechtlichen Sprache/Kommunikation, Rechts- und Verfahrenskenntnisse. Dabei handelt es sich in der Regel um Männer, gelegentlich werden aber auch Frauen von Amtsträgern oder Hebammen als Akteure infrajustizieller Konfliktregulierung ausgemacht. ${ }^{17}$

Sozial verorten lassen sich die Akteure der Infrajustiz besonders in den lokalen Eliten (die notables $d u$ village) oder als Funktionsträger korporativer/kommunaler Organe. Sie übten kein professionelles Amt in staatlichen Gerichten aus, konnten aber auch eine Art Zwischenstellung einnehmen, wie z.B. Friedensrichter, justices of the peace, Amtleute, Schreiber, Geistliche oder Notare. ${ }^{18}$ Besonders die letzteren hat die französische For-

17 Ulrike Gleixner, „Das Mensch“ und „,der Kerl“. Die Konstruktion von Geschlecht in Unzuchtsverfahren der Frühen Neuzeit (1700-1760), Frankfurt a.M. u.a. 1994; Heinrich Richard Schmidt, Dorf und Religion. Reformierte Sittenzucht in Berner Landgemeinden der Frühen Neuzeit, Stuttgart u.a. 1995; Katharina Simon-Muscheid, Frauen vor Gericht. Erfahrungen, Strategien und Wissen, in: Münch, Erfahrung, S. 389-399.

18 Peter Wettmann, „Arbitrator“ und „Adjudicator“: Zur Bedeutung der englischen Justices of the Peace bei der formellen und informellen Konfliktregelung in ländlichen Gemeinden des 18. Jahrhunderts, in: Kriminologisches Journal, Beiheft 2 (1987): Kriminologie und Geschichte, hrsg. von Gerlinda Smaus, S. 111-130; Norma Landau, The Justices of the Peace, 1679-1760, Berkely 1984; Jacques-Guy Petit (Hrsg.), Une justice de proximité. La justice de paix (1790 - 1958), Paris 2003; zur Geistlichkeit vgl. z.B. Andreas Holzem, Katholische Konfession und Kirchenzucht. Handlungsformen und Deliktfelder archidiakonaler Gerichtsbarkeit im 17. und 18. Jahrhundert, in: Westfälische Forschungen 45 (1995), S. 295-332. 
schung als Akteure der Infrajustiz intensiver erforscht. ${ }^{19}$ Betont werden als Motive der Vermittler deren Eigeninteressen an Infrajustiz insbesondere die Zunahme von sozialem Prestige bzw. sozialem Kapital, Macht, Einfluss und Beziehungen sowie auch finanzielle/materielle Profite. Diese spezifischen Akteure bzw. ihre besonderen Merkmale verschwinden zwar nicht im 19. Jahrhundert mit der bürgerlichen Gesellschaft und dem modernen Staat, aber sie verlieren ihre Exklusivität und durch das moderne Beamtentum z.T. ihre Zwischenstellung.

\section{Verfahren/Modi}

Die Verfahren und Modi der infrajustiziellen Konfliktregulierung umfassen eine große Bandbreite an Praktiken, die sowohl der Sphäre autonomer sozialer Konfliktregulierung als auch derjenigen von Recht, Gericht und Verwaltung zugeordnet werden können. Für das vormoderne Rechtssystem gilt dabei gerade für den Bereich von Devianz/Kriminalität, dass es nicht als völlig verstaatlicht und abgeschlossen gelten kann, sondern durch Rechtspluralismus, zahlreiche nicht-staatliche Institutionen und Akteure auf der lokalen und niedergerichtlichen Ebene und eine entsprechende Vielfalt an Verfahren gekennzeichnet war, die von traditionellen Frevel- und Rügeverfahren über summarische policeyliche oder niedergerichtliche Verfahren bis zum dualen (in ein lokales Untersuchungs- und zentrales Entscheidungsverfahren geteilten) Inquisitionsprozess reichten. ${ }^{20}$ Grundsätzlich geht die Forschung zwar von interpersonalen Kommunikationsvorgängen zwischen Konfliktparteien und Vermittlern aus, aber darüber hinaus spielen auch die Öffentlichkeit sowie rechtlich-schriftliche Kommunikationsformen und die Interaktion mit der Obrigkeit eine wesentliche Rolle. Angenommen werden alternative multiple Handlungsoptionen, die einzelne Abschnitte des informellen Konfliktregulierungsverfahrens - Einleitung, Aushandeln, Ergebnis - betreffen und sich zwischen accommodement und vengeance privée bewegen. Wesentlich hierbei ist auch das Zusammenspiel bzw. das Einbringen infrajustizieller und ,außergerichtlicher" Modi in das förmliche Strafverfahren über letztlich ,außergerichtliche“ Elemente wie Dispensgesuche, Bittschriften, Supplikationen oder Gnadenbitten. ${ }^{21}$

Im Hinblick auf die Initiierungsphase werden Gerede/Gerücht, Denunziation/Anzeige bei Amtspersonen und die Drohung mit der Einschaltung eines Gerichts genannt; Kommunikationsmittel bilden dabei auch Supplikation oder die Lettres de cachet. Die Phase der informellen Verhandlungen ist geprägt durch Vermittlung, Schlichtung, Beratung und Aushandeln. Das Ergebnis manifestiert sich in einem privaten oder zumindest

19 Soman, L'infra-justice; Stéphane Trayaud, Notariat et infrajustice. Le rôle de médiation du notaire sous l'Ancien Régime à travers la pratique de Pierre Thoumas de Bosmie, notaire royal à Limoges (1735-1740), in: Revue d'histoire de l'enfance «irrégulière» (2001), S. 207-220.

20 Karl Härter, Strafverfahren im frühneuzeitlichen Territorialstaat: Inquisition, Entscheidungsfindung, Supplikation, in: Blauert/Schwerhoff, Kriminalitätsgeschichte, S. 459-480.

21 Farge/Foucault, Familiäre Konflikte; Nubola/Würgler, Suppliche e „gravamina“; Härter/ Nubola, Grazia e giustizia; Ulrike Ludwig, Das Herz der Justitia. Gestaltungspotentiale territorialer Herrschaft in der Strafrechts- und Gnadenpraxis am Beispiel Kursachsens 1548-1648, Konstanz 2008. 
teilöffentlichen Raum (reconnaissance publique) als Ausgleich, förmlicher, teils ritualisierter und öffentlicher Versöhnung (réconciliation en publique), dem Verzicht auf soziale Sanktionen oder Rache, der Wiederherstellung der Ehre und materiellen Kompensationsleistungen, fixiert in Abkommen und Verträgen oder auch durch mündliche und öffentliche Rituale bekräftigt. Zu diesen Praktiken, die sich insbesondere bei Ehrund Gewaltkonflikten bzw. den entsprechenden Delikten auch in der Justizpraxis nachweisen lassen, gehören Sühneverträge, öffentliche Eidesleistungen (juramentum den non offendo) oder die öffentliche Abbitte, teilweise mit Ritualen wie dem Handschütteln oder Ehrenerklärungen verbunden. Im Rahmen eines Strafverfahrens konnten zudem private Kompensationen und Ausgleichzahlungen verlangt oder mitreguliert werden, wobei dies häufig mit Verzicht auf öffentliche Strafe gekoppelt war. Die zeitgenössische Jurisprudenz diskutierte solche noch im 18. Jahrhundert in den meisten europäischen Rechtssystemen üblichen Praktiken als herrschaftliche oder fiskalische Composition oder Vergleichung, aus der sich im 19. Jahrhundert die Figur der Privatstrafe bzw. des Strafschadenersatzes entwickelte, die eine „Verrechtlichung“ und „Verstaatlichung“" infrajustizieller Praktiken markieren. ${ }^{22}$ Kam es zu keiner Konfliktlösung, standen den Betroffenen soziale Sanktionen (Schmähen, Verrufen, Schandbriefe usw.), ${ }^{23}$ Rache oder die Einschaltung der Justiz zur Verfügung. Letztere wird meist als Justiznutzung konzeptualisiert, wobei die Justiz als End- oder Ausgangspunkt einer Konfliktbearbeitung im infrajustiziellen Raum fungieren konnte, je ob Anzeige oder Klageverzicht benutzt wurden. ${ }^{24}$

Die Verbrechen, welche die Gerichte verhandelten, stehen insofern stellvertretend für teils anders gelagerte soziale Konflikte. Darüber hinaus konnten die Gerichte aber auch auf Veranlassung oder selbsttätig eine quasi infrajustizielle Regulierung außerhalb des „ordentlichen“ Verfahrens bzw. der Funktionen Entscheidung und Strafe vornehmen: Beispiele hierfür wären die recognizanses in England, die den Konfliktparteien eine

22 Friedrich Schaffstein, Wiedergutmachung und Genugtuung im Strafprozeß vom 16. bis zum Ausgang des 18. Jahrhunderts, in: Heinz Schöch (Hrsg.), Wiedergutmachung und Strafrecht, München 1987, S. 9-27; Heiner Lück, Sühne und Strafgerichtsbarkeit im Kursachsen des 15. und 16. Jahrhunderts, in: Hans Schlosser/Dietmar Willoweit (Hrsg.), Neue Wege strafrechtsgeschichtlicher Forschung, Köln u.a. 1999, S. 83-99; Laurence Montazel, La peine privée en France et Allemagne (XVIème - XIXème siècles). Essai sur une notion doctrinale commune, in: Ius commune 28 (2001), S. 161-202; Esther Sonntag, Entwicklungstendenzen der Privatstrafen. Strafschadensersatz im antiken römischen, deutschen und US-amerikanischen Recht, Berlin 2005.

23 Matthias Lentz, Schmähbriefe und Schandbilder als Medien außergerichtlicher Konfliktbewältigung. Von der sozialen Sanktion zur öffentlichen Strafe (14. - 16. Jahrhundert), in: Schlosser/Willoweit, Neue Wege, S. 55-81.

24 Martin Dinges, Frühneuzeitliche Justiz: Justizphantasien als Justiznutzung am Beispiel von Klagen bei der Pariser Polizei im 18. Jahrhundert, in: Heinz Mohnhaupt/Dieter Simon (Hrsg.), Vorträge zur Justizforschung. Geschichte und Theorie, Bd. 1, Frankfurt a.M. 1992, S. 269-292; Martin Dinges, Michel Foucault, Justizphantasien und die Macht, in: Andreas Blauert/Gerd Schwerhoff(Hrsg.), Mit den Waffen der Justiz. Zur Kriminalitätsgeschichte des Spätmittelalters und der Frühen Neuzeit, Frankfurt a.M. 1993, S. 189-212; Martin Dinges, Justiznutzungen als soziale Kontrolle, in: Blauert/Schwerhoff, Kriminalitätsforschung, S. 503-544. 
außergerichtliche Kompensation (und Strafe bei Nichterfüllung) auferlegten, ${ }^{25}$ oder Dispens und Gnade, erwirkt z.B. durch außergerichtliche Supplikationen. Auch die Konfliktregulierung im Binnenraum der Strafjustiz konnte infrajustiziellen Modi und Zwecken folgen und ,private“ Kompensation, Ausgleich und Frieden anstelle staatlicher Strafe präferieren. Insofern weisen auch Verfahren und Modi im Konzept der Infrajustiz einen Rechtsbezug auf. Der Grad der Verbindlichkeit der infrajustiziellen Praktiken wie die zugrundliegenden Regeln sind allerdings kaum näher theoretisch bestimmt, sondern werden in der Forschung meist lediglich anhand - teils differenter Beispiele empirisch dargestellt. ${ }^{26}$

\section{Normen, Ordnungsmodelle, Funktionen}

Wie meist generell in der historischen Kriminalitätsforschung geht auch das Modell der Infrajustiz von einem vormodernen Normenpluralismus aus, d.h. neben dem obrigkeitlich-staatlichen Recht werden soziale und sonstige Normen vorausgesetzt, die in der infrajustiziellen Konfliktregulierung zugrunde gelegt wurden. Neben „Normenpluralismus" fungiert das von Keith Wright postulierte concept of two orders gelegentlich als theoretische Beschreibung; andere Autoren sprechen von unwritten law, das z.B. in Kent noch im 19. Jahrhundert wesentlich außer- wie auch gerichtliche Konfliktregulierung geprägt habe. ${ }^{27}$ Im Fall religiöser oder genossenschaftlicher Normen lassen sich normative Ordnungsmodelle der Infrajustiz noch relativ genau nachvollziehen; meist werden sie aber lediglich aus der infrajustiziellen Praxis selbst gefolgert und nicht näher bestimmt.

Im Hinblick auf die Interessen und Ziele der infrajustiziellen Konfliktregulierung betont die Forschung vor allem Frieden und Friedensstiftung sowie Kompensation und Ausgleich. Der außergerichtliche Interessenausgleich konnte materiell wie auch immateriell als Wiederherstellung von Ehre erfolgen. Damit verbindet sich eine insgesamt positive Darstellung der Effektivität oder Wirkung von Infrajustiz im Hinblick auf Gewaltvermeidung bzw. Pazifizierung, Konfliktlösung und sozialen Frieden. ${ }^{28}$ „Vergeltung“ im Sinne von „Rache“ und gewaltsame Modi der Konfliktregulierung werden

25 G. T. Smith, Violent crime and the public weal in England, 1700-1900, in: R. Mc Mahon (Hrsg.), Crime, Law and Popular Culture in Europe, 1500-1900, Cullompton 2008, S. 190-218, hier S. 205.

26 Vgl. zur Kritik Garnot, Justice, infrajustice, parajustice, S. 112 ff.; Loetz, L'infrajudiciaire, S. $561 \mathrm{f}$.

27 Keith Wrightson, Two concepts of order. Justices, constables and jurymen in seventeenthcentury England, in: John Brewer/John Styles (Hrsg.), An Ungovernable People. The English and their Law in the $17^{\text {th }}$ and $18^{\text {th }}$ Centuries, London 1980, S. 21-46; Carolyn A. Conley, The Unwritten Law. Criminal Justice in Victorian Kent, Oxford 1991; allgemein: Schwerhoff, Kriminalitätsforschung, S. $74 \mathrm{ff}$.

28 Benoît Garnot, Conclusion, in: Garnot, L'infrajudiciaire, S. 467-471; Loetz, L'infrajudiciaire, S. $558 \mathrm{ff}$. 
lediglich im Zusammenhang mit Fehde und Duell als violence préalable angesprochen, sind jedoch theoretisch eher unzureichend in das Konzept integriert. ${ }^{29}$

Bezüglich Motiven und Interessen der Konfliktparteien wird übereinstimmend betont, dass infrajustizielle Konfliktregulierung stärker den Opferinteressen entgegenkam und als weniger kostenintensive, günstigere, flexiblere und effektivere Konfliktregulierung gelten könne. Aushandeln, Mediation, Arrangement und Kompensation erbringen einen profit de la réparation, der durch staatliche Strafjustiz und Strafe nicht erreicht werde; für die zahlende/unterlegene Partei bestünde dieser besonders in der Strafvermeidung. ${ }^{30}$

Konfliktparteien nutzten den Weg zur Justiz daher auch als Druckmittel, um Konfliktlösung im infrajustiziellen Raum zu erreichen, denn eine Schwäche infrajustizieller Lösungen bestand in der Durchsetzbarkeit. In dieser Beziehung fungiert Infrajustiz als Vorstufe zur Justiz, die als Option zur Verfügung stand und eingeschaltet werden konnte, sobald der Konflikt keiner außergerichtlichen Lösung mehr zugeführt werden konnte. Offen bleibt dabei, ob danach für die Konfliktparteien noch ein dropout möglich war, folgte die Strafjustiz doch eigenen Systemlogiken und Rationalitäten. Ein Konflikt, der zu einem Verbrechen transformiert und in die Justiz eingespeist wurde (auch von den Betroffenen selbst) konnte gerade unter den Bedingungen des ius commune und des Inquisitionsprozesses einen völlig anderen Verlauf nehmen und zu für beide Konfliktparteien unerwünschten Ergebnissen (Strafe, Kosten, geringe oder keine Kompensation) führen. ${ }^{31}$

Wesentlich ist folglich die Frage nach der Autonomie und dem Funktionszusammenhang: Bezeichnet Infrajustiz einen Bereich weitgehend autonomer Konfliktregulierung, um die „,verhasste“ Justiz zu vermeiden? Oder handelt es sich um eine komplementäre Alternative zur obrigkeitlich-staatlichen Justiz, in der Gerichte von den Konfliktparteien als eine zusätzliche Option ins Spiel gebracht wurden? Francisca Loetz hat argumentiert, dass die Zeitgenossen die außergerichtlichen Vermittlungsversuche als Vorstufe der Justiz angesehen hätten, und auch die französische Forschung hat den Zusammenhang und die Komplementarität zur staatlichen Justiz betont. ${ }^{32}$ Justiz und Infrajustiz bilden ein komplexes (teilweise komplementäres) Gesamtsystem von Konfliktregulierung, das auf die Erhaltung/Herstellung von sozialem Frieden und sozialer Ordnung zielte. Insofern konnte der Staat aus utilitaristischen Gründen infrajustizielle Konfliktregulierung als eine Alternative durchaus tolerieren, akzeptieren oder sogar partiell in das Rechtssystem integrieren.

29 Garnot, Conclusion, S. 468: "la violence préalable est parfois nécessaire; le duel, la vengeance, le lynchage, peuvent être des modes de régulation permettant un règlement pacifique ultérieur".

30 Farcy, Justice privée et Justice publique, S. 11; Garnot, Justice, infrajustice, parajustice; Loetz, L'infrajudiciaire, S. 562.

31 So jedenfalls das Ergebnis meiner Fallstudie: Härter, Policey und Strafjustiz.

32 Loetz, L'infrajudiciaire, S. 555; Garnot, Conclusion, S. 468: „L'infrajudiciaire ne se conçoit donc pas sans l'existence du judiciaire, parce qu'il occupe la place laissée libre par celui-ci (et même au-delà, nous l'avons vu). En effet, son ampleur dépend, pour beaucoup, de l'ampleur même du judiciaire, même si les rapports entre les deux secteurs sont ambigus". Noch stärker betont in: Garnot, Justice, infrajustice, parajustice. 
Andere wie Martin Dinges haben dagegen die Bedeutung staatlicher Justiz negiert und weitgehende Autonomie und Handlungsfreiheit der Konfliktparteien im Rahmen der Infrajustiz postuliert. Infrajustizielle Konfliktregulierung sei grundsätzlich präferiert worden, da die Konfliktparteien die staatliche Justiz abgelehnt oder ihr misstraut hätten. ${ }^{33}$ Infrajustiz würde damit vor allem Justizvermeidung bedeuten bzw. beschreiben. Die Infrajustiz und ihre Akteure bedienten sich lediglich der passiven staatlichen Justiz, unterwanderten diese und formierten ein eigenes autonomes ,alternativ-substitutives Rechtssystem“, das konkurrierte oder „Widerstand“ leistete.

Als Argument dafür, dass dies im Bereich der Strafjustiz möglich sei, wird meist die institutionelle Schwäche des vormodernen Staates angeführt, der seine Normen nicht durchsetzen konnte oder wollte. Auch wenn ich diese Deutung ablehne, so lässt sich dennoch zeigen, dass der vormoderne Staat infrajustizielle Praktiken zuließ und sich dieser sogar bediente, um institutionelle/exekutive Schwächen zu kompensieren und Kosten zu vermeiden - ein Muster, das auch heute noch eine Rolle spielen mag. Insofern war Infrajustiz aber auch partiell staatlich toleriert oder gar legitimiert und bedeutete keineswegs völlige Autonomie und völlige Handlungsfreiheit der Konfliktparteien im Hinblick auf Konfliktregulierung. Zudem baute der Staat seit der Frühen Neuzeit die Normierung, Kontrolle und Inklusion infrajustizieller Akteure/Institutionen und Verfahren aus oder ,,verstaatlichte“ diese. ${ }^{34}$

\section{Schluss}

Das Phänomen der Infrajustiz bzw. der Konfliktregulierung im Umfeld von Gerichten oder in der Interaktion außergerichtlicher Akteure mit obrigkeitlich-staatlicher Strafjustiz ist in vielen Fallstudien der historischen Kriminalitätsforschung empirisch nachgewiesen. Allerdings geben meist nur obrigkeitliche Gerichtsquellen und Akten Einblick in die infrajustizielle Praxis, die damit oft im Dunkel nicht überlieferter Interaktionen bleibt. Dies mag auch ein Grund dafür sein, dass sie letztlich noch nicht zu einem konsistenten, widerspruchsfreien theoretischen Modell entwickelt wurde. Defizite, Wi-

33 Dinges, Négocier son honneur; Zusammenfassung der Positionen bei Garnot, Justice, infrajustice, parajustice, S. $104 \mathrm{ff}$.

34 So jedenfalls das Ergebnis meiner Forschungen: Härter, Policey und Strafjustiz; Karl Härter, Strafen mit und neben der Zentralgewalt: Pluralität und Verstaatlichung des Strafens in der Frühen Neuzeit, in: Günther Schlee/Bertram Turner (Hrsg.), Vergeltung. Eine interdisziplinäre Betrachtung der Rechtfertigung und Regulation von Gewalt, Frankfurt a.M./New York 2008, S. 105-126; Karl Härter, Die Entwicklung des Strafrechts in Mitteleuropa 1770-1848: Defensive Modernisierung, Kontinuitäten und Wandel der Rahmenbedingungen, in: Habermas/Schwerhoff, Verbrechen im Blick, S. 71-107. Vgl. aber zur kontroversen französischen Debatte im Kontext der Infrajustice: Jacques-Guy Petit, La Justice en France, 1789-1939. Une étatisation modèle?, in: Crime, Histoire \& Sociétés/Crime, History \& Societies 6 (2002), S. 85-103; Chauvaud Frédéric, La Justice en France, 1789-1939. Un modèle à l'épreuve, in: ebd., S. 105-125; Farcy, Justice privée et Justice publique. 
dersprüche und offene Fragen lassen sich daher durchaus benennen: ${ }^{35}$ So bleiben Spielräume und Auswahlfreiheit zwischen dem Gang zum Gericht und infrajustiziellen Optionen der Konfliktregulierung oft unbestimmt; dies gilt auch für den Zugang zu den jeweiligen Möglichkeiten, die Frage der Chancengleichheit oder die Motivation von Konfliktpartnern zur Teilnahme an infrajustiziellen Praktiken. Zu berücksichtigen ist ebenfalls, dass Staat und Rechtssystem die jeweiligen Rahmenbedingungen und damit Optionen und Auswahl beeinflussen konnten. Das Modell der Infrajustiz muss daher im Hinblick auf historischen Wandel die allmähliche Durchsetzung einer staatlichen Strafverfolgungspflicht und eines entsprechenden Instrumentariums der sozialen Kontrolle mit einbeziehen und kann die obrigkeitlich-repressive Seite der Strafjustiz nicht völlig außer Acht lassen. Auch die Um- und Durchsetzungschancen infrajustizieller Konfliktregulierung lassen sich nur schwer systematisch erfassen. War das Ergebnis immer die Befolgung und Einhaltung infrajustizieller Agreements, nachhaltige Konfliktlösung und sozialer Frieden? Ungelöste Konflikte, Konfliktverschärfung und Gewalt werden im Rahmen der Infrajustiz kaum berücksichtigt und theoretisch nur unzureichend konzeptualisiert. Insofern bedarf auch die damit in Verbindung stehende Frage der Rechtssicherheit bzw. einer nachhaltigen, akzeptierten und legitimen Konfliktlösung, die der Infrajustiz - meist unkritisch - zugebilligt wird, einer kritischen Würdigung, und zwar gerade im Hinblick auf die Konfliktparteien und Akteure. ${ }^{36}$

Zwar kann die Forschung infrajustizielle Konfliktregulierung zwischen diesen ,privaten Akteuren“ für viele Konfliktformen belegen, eine systematische Typologie fehlt aber bislang, und häufig werden Konfliktfelder, die kaum im infrajustiziellen Bereich verhandelt wurden, außer Acht gelassen. Devianz und Kriminalität lassen sich aber vor allem auch als Normkonflikte bzw. Konflikte zwischen Normbrechern und dem Rechtssystem/Staat lesen, die im Binnenraum der Strafjustiz - durchaus auch mit infrajustiziellen Praktiken - bearbeitet werden konnten. Insofern wäre auch dieser Binnenraum stärker einzubeziehen und weniger von der Dichotomie außergerichtlich - gerichtlich als vielmehr von infrajustiziellen Verfahren, Konflikten und Akteuren auszugehen, die im Justizsystem und seinem infrajustiziellen Umfeld Relevanz besitzen oder gewinnen konnten. Gewichtung und Verhältnis der Konfliktregulierung zwischen obrigkeitlichstaatlicher Justiz, Infrajustiz, sozial autonomer Regulierung und Nicht-Behandlung bleiben somit noch teilweise diffus. Infrajustiz kann ebenfalls nur einen Teil außergerichtlicher Konfliktregulierung beschreiben, und ein wesentlicher Teil von Kriminalität und Konflikt verbleibt in einem - schon aufgrund der Quellenlage - historisch nur schwer fassbaren Bereich autonomer sozialer Selbstregulierung oder wurden keiner Bearbeitung zugeführt und toleriert. Letzteres hat Garnot mit dem eher nebulösen Begriff der extra-justice zu beschreiben versucht, die er von der Parajustiz des infrajusti-

35 Vgl. auch die kritischen Punkte bei Benoît Garnot, L' ampleur et les limites de l'infrajudiciaire dans la France d'Ancien Régime (XVIe, XVIIe et XVIIIe siècle), in: Garnot, L'infrajudiciaire, S. 69-76; Jean-Claude Farcy, Peut-on mesurer l'infrajudiciaire?, in: ebd. S. 109-127; Garnot, Conclusion; Garnot, Justice, infrajustice, parajustice; Loetz, L'infrajudiciaire; Farcy, Justice privée et Justice publique.

36 Hierzu demnächst: Karl Härter, Die Sicherheit des Rechts und die Produktion von Sicherheit im frühneuzeitlichen Strafrecht, in: Christoph Kampmann/Ulrich Niggemann (Hrsg.), Sicherheit in der Frühen Neuzeit [im Druck]. 
ziellen Raums unterscheidet. ${ }^{37}$ Das Feld der außergerichtlichen Konfliktregulierung, das keinen oder nur geringen Bezug zum Recht aufweist, wird letztlich auch vom Konzept der Infrajustiz theoretisch und empirisch kaum erfasst.

Das Verhältnis von nicht bearbeiteten Konflikten, autonomer sozialer Selbstregulierung, Infrajustiz und staatlicher Justiz stellt gerade im Hinblick auf die langfristige historische Entwicklung ein Problem dar. Die Vertreter der Infrajustiz lehnen lineare Modernisierungsmodelle $a b$ und bestehen auf sich jeweils ausformende unterschiedliche „Mischungsverhältnisse“. ${ }^{38}$ Die allmähliche Ausformung eines staatlichen Justiz- und Gewaltmonopols seit dem 19. Jahrhundert, der processus d'acculturation judiciaire des populations, die Kriminalisierung außergerichtlicher Konfliktregulierung (wie Fehde und Duell) und die elimination der justice privee sowie die Verstaatlichung intermediärer, genossenschaftlicher, kommunaler Formen von Konfliktregulierung und Justiz und deren Professionalisierung können aber kaum negiert werden und sie beeinflussten infrajustizielle Optionen und Praktiken. ${ }^{39}$ So konstatiert Rebekka Habermas ab Mitte des 19. Jahrhunderts bei Eigentumsdelikten eine zunehmende Nutzung der Gerichte durch die ländliche Bevölkerung, um damit Interessen- und Ehrkonflikte zu artikulieren und zu regulieren. Grund hierfür seien aber weniger die Justizreformen, sondern die Veränderungen der Eigentums- und Ehrkonzepte, die zu einer Verlagerung aus dem infrajustiziellen Raum in das Gericht geführt hätten. ${ }^{40}$ Ergänzend wäre hinzuzufügen, dass z.B. im Hinblick auf Gewalt und Ehre das moderne „Rechtssystem“ durchaus infrajustizielle Modi und Elemente im 19. Jahrhundert adaptierte und integrierte. ${ }^{41}$ Diesbezüglich nennt Farcy für Frankreich exemplarisch die ,justice de paix avec son bureau de paix et de conciliation, tribunaux des famille, arbitrage étendu à nombreuses matières". ${ }^{42}$ Die Verrechtlichung und Integration - inklusive der Kriminalisierung und Eliminierung - infrajustizieller Konfliktregulierung bilden folglich ein lohnenswertes Feld, um der Infrajustiz auch in der Moderne weiter nachzugehen; als Stichworte seien hier nur Modelle genannt wie court shopping, plea-bargaining, restorative justice, Kompensation oder Mediation. ${ }^{43}$

Insofern kann das Konzept der „Infrajustiz“ trotz der skizzierten theoretischen Defizite eine Funktion gewinnen, um empirisch feststellbare Phänomene infrajustizieller

37 Garnot, Justice, infrajustice, parajustice; vgl. Farcy, Justice privée et Justice publique, S. 15: „Enfin, une criminalité non régulée, subie ou tolérée, sans règlement du conflit rentrerait dans le cadre de l'extra-justice“".

38 Vgl. Loetz, L'infrajudiciaire, S. $560 \mathrm{f}$.

39 Farcy, Justice privée et Justice publique, S. 11 ff.; Härter, Strafen mit und neben der Zentralgewalt; Härter, Entwicklung des Strafrechts.

40 Rebekka Habermas, Diebe vor Gericht. Die Entstehung der modernen Rechtsordnung im 19. Jahrhundert, Frankfurt a.M. 2008.

41 Härter, Violent Crimes and Retaliation.

42 Farcy, Justice privée et Justice publique, S. 18.

43 Aus der abundanten Literatur vgl. exemplarisch Neil Andrews, The three paths of justice. Court proceedings, arbitration, and mediation in England, New York 2012, und mit rechtshistorischem Bezug: Thomas Feltes, Die außergerichtliche Erledigung von Konflikten. Historische Aspekte und aktuelle Bezüge, in: Heinz Mohnhaupt/Dieter Simon (Hrsg.), Vorträge zur Justizforschung. Geschichte und Theorie, Bd. 2, Frankfurt a.M. 1993, S. 581-597; Wolf Heydebrand, Neue Formen der Justiz in den USA zwischen formellem Prozeß, Verhandeln und Vergleich, in: ebd. S. 599-625. 
Konfliktregulierung unter Mitwirkung Dritter im Umfeld wie im Binnenraum der Justiz zu beschreiben und Fragestellungen der außergerichtlichen-gerichtlichen Konfliktlösung zu bearbeiten. ${ }^{44}$ Aus der Perspektive von Devianz/Kriminalität und den zugrunde liegenden Konflikten erscheint freilich die das positive Ergebnis betonende „Konfliktlösung“" als problematisch und wäre durch Konfliktbearbeitung oder Konfliktregulierung zu ersetzen, die Akteure, Optionen, Handlungen und das Prozesshafte betonen. Weiterhin kann das Konzept der Infrajustiz helfen, eine zu rigide Unterscheidung von außergerichtlich und gerichtlich zu überwinden, die eine Dichotomie des EntwederOder impliziert. Denn Infrajustiz betont die Interaktionen und Interdependenzen zwischen obrigkeitlich-staatlicher Justiz und außergerichtlicher Konfliktregulierung, wobei letztere kaum als völlig autonom konstruiert werden kann, sondern (rechts-)historisch im Umfeld oder auch im Binnenraum des Rechts stärker zu erforschen wäre. Insofern lässt sich Infrajustiz gut mit anderen, in der historischen Kriminalitätsforschung verwendeten Konzepten wie Justiznutzung oder (miteinander verschränkter formeller/vertikaler und informeller/horizontaler) sozialer Kontrolle verbinden. Offen bleibt die Frage der Verrechtlichung: Infrajustiz bildet zwar kein Gegenmodell zum Prozess der Verrechtlichung von Konflikten, sondern könnte sich als ein Feld oder ein Modus begreifen lassen. Dennoch stellt Verrechtlichung im Sinne einer langfristigen Zunahme gerichtlicher Konfliktregulierung Infrajustiz auch in Frage, falls deren Normen und Verfahren als „nicht-rechtlich“ aufgefasst werden. Der Rechtscharakter von Infrajustiz bzw. außergerichtlicher Konfliktregulierung bedarf jedenfalls in der historischen Dimension weiterer Diskussion. Bezüglich der Strafjustiz kann Infrajustiz aber in Verbindung mit Konzepten wie Justiznutzung und sozialer Kontrolle eine gewisse Engführung und letztlich auch theoretische Unschärfe des Ansatzes ,,außergerichtliche und gerichtliche Konfliktlösung“ überwinden helfen. Dies wird jedoch ebenso wie die Frage einer schärferen theoretischen Konzeptualisierung der Infrajustiz weiter zu diskutieren sein.

44 J. A. Sharpe in seiner Rezension zu Garnot, L'Infrajudiciaire, in: Crime, Histoire \& Sociétés/ Crime, History \& Societies 5 (2001), S. 140-142 charakterisiert das Modell der Infrajustiz als „indispensable research tool for the historian of the law and the operation of legal systems“. 